\title{
Positive Regulation of Platelet Aggregation Process
}

National Cancer Institute

\section{Source}

National Cancer Institute. Positive Regulation of Platelet Aggregation Process. NCI

Thesaurus. Code C40712.

Any platelet aggregation process that increases the rate, frequency or extent of platelet aggregation. 